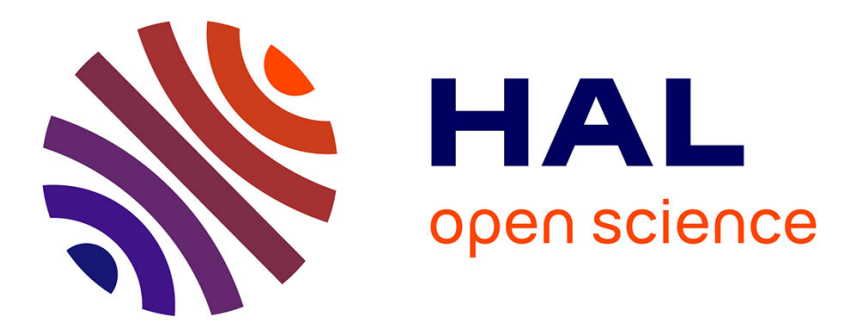

\title{
Complexity bounds for obstacle avoidance within a zonotopic framework
}

Daniel Ioan, Ionela Prodan, Florin Stoican, Sorin Olaru, Silviu-Iulian

Niculescu

\section{- To cite this version:}

Daniel Ioan, Ionela Prodan, Florin Stoican, Sorin Olaru, Silviu-Iulian Niculescu. Complexity bounds for obstacle avoidance within a zonotopic framework. [Research Report] L2S, CNRS, Supelec, Université Paris Sud. 2019. hal-01986337

\section{HAL Id: hal-01986337 \\ https://hal-centralesupelec.archives-ouvertes.fr/hal-01986337}

Submitted on 18 Jan 2019

HAL is a multi-disciplinary open access archive for the deposit and dissemination of scientific research documents, whether they are published or not. The documents may come from teaching and research institutions in France or abroad, or from public or private research centers.
L'archive ouverte pluridisciplinaire HAL, est destinée au dépôt et à la diffusion de documents scientifiques de niveau recherche, publiés ou non, émanant des établissements d'enseignement et de recherche français ou étrangers, des laboratoires publics ou privés. 


\title{
Complexity bounds for obstacle avoidance within a zonotopic framework
}

\author{
Daniel Ioan ${ }^{1}$, Ionela Prodan ${ }^{2}$, Florin Stoican ${ }^{3}$, Sorin Olaru ${ }^{1}$, Silviu-Iulian Niculescu ${ }^{1}$
}

\begin{abstract}
This paper addresses the problem of collision avoidance in a multi-obstacle environment and focuses on its representation in optimization-based control problems. The design problem is commonly stated in the literature in terms of a constrained optimization problem over a non-convex domain. Describing this non-convex domain has computational and structural implications, generally leading to a trade-off between computational efficiency and control performance. Preliminary results make use of hyperplane arrangements to characterize these regions. The hyperplane arrangements build on generic polyhedral sets and a natural question is whether additional structure could bring a benefit. The current paper addresses this issue by the use of zonotopic over-approximation and highlights their benefits when introduced in the obstacle avoidance problem. Comparisons with classical sampled-based approaches are presented through simulations.
\end{abstract}

\section{INTRODUCTION}

Recent advances in the field of multi-agent dynamical systems show that an adequate collision avoidance strategy is fundamental in order to ensure the safety and the integrality of the system and of the environment. In the recent years, the control community has endeavored to obtain collisionfree control strategies which permit real-time operations [1].

There are two main approaches in the literature which tackle the collision avoidance problem: direct and indirect methods. The former take explicitly into account the constraints and lead to a constrained optimization problem which often reduces to MI (mixed-integer) formulations [2], [3]. The later relax the constraints by adding penalty terms in the cost, e.g., potential field-based formulations [4], [5]. The direct methods are considered more robust at the price of higher computational loads. Other approaches dealing with the path/trajectory planning in a non-convex feasible space are the sampled-based methods, such as PRM (probabilistic roadmaps) [6] or RRT (rapidly-exploring random tree) [7]. These replace the search of feasible paths with the search of the shortest (w.r.t. a predefined criterion) path within a graph whose nodes are randomly selected samples [8].

Often the obstacles are modeled as convex polytopic regions in which case the resulting non-convex and nonconnected feasible domain can be efficiently described through its associated hyperplane arrangement [9] which allows to characterize the domain as a union of convex cells.

\footnotetext{
${ }^{1}$ Laboratory of Signals and Systems, Univ. Paris-Sud-CentraleSupelecCNRS, Université Paris Saclay. \{daniel.ioan, sorin.olaru, silviu.niculescu\}@l2s. centralesupelec.fr

${ }^{2}$ Univ. Grenoble Alpes, Grenoble INP, LCIS, ionela.prodan@lcis.grenoble-inp. fr

${ }^{3}$ Department of Automatic Control and Systems Engineering, UPB, Romania florin.stoicandacse.pub.ro
}

Next, a mixed-integer formulation is used to characterize the union of cells and is introduced in a standard constrained optimization problem. Technical procedures like cell merging [10] or logarithmic formulations [11] are employed to reduce the complexity of the formulation but their automatic treatment becomes numerically complex for large numbers of obstacles and/or agents and this will represent the focus of the present paper.

In a nutshell, the question to be addressed is: "Can one approximate the obstacles such that a lower number of cells describing the feasible domain is obtained while in the same time safeguarding the features of the initial problem?". We propose to address this question by considering zonotopes and their properties for obstacle over-approximation and cell counting while providing an explicit measure of the problem complexity in terms of the total number of cells. Due to their intrinsic properties, zonotopes (genuine or as intervals [12]) are widely used in control, e.g., for collision detection [13], reachability analysis [14], fault diagnosis [15] or guaranteed state estimation [16]. Moreover, there are toolboxes like CORA [17] able to manipulate and display these zonotopic sets representations.

We start from exact formulations (set inclusion constraints, analytic formulation of the zonotope's volume, etc.) and relax them to a linear constrained form (which depends on a collection of centers and scaling factors).

The main contributions of this paper are the following:

i) provide tight zonotopic approximations of the obstacles;

ii) provide a rigorous bound for the number of generators such that the complexity is reduced;

iii) propose various measures for zonotopic approximation;

iv) provide comparisons between MI formulation of the collision avoidance problem and a classical sampledbased method (PRM).

The remaining of this paper is divided as follows: Section II presents some basic set-theoretic tools and the related control problems and Section III introduces the formulation of optimized zonotopic over-approximation problem. Furthermore, Section IV treats the approximation from the control perspective and evaluates the result on different test scenarios while Section V draws the conclusions.

Notation: The Minkowski sum of two sets is denoted as $A \oplus B=\{x: x=a+b, a \in A, b \in B\} . \mathcal{C}_{X}(S)$ denotes the complement of the set $S$ over $X \in \mathbb{R}^{d}$. Any polytope (i.e. a bounded polyhedron) has a dual representation in terms of intersection of half-spaces or convex hull of extreme points: $P=\left\{x: s_{i}^{\top} x \leq r_{i}, \forall i\right\}=\left\{x: x=\sum \alpha_{j} v_{j}, \sum \alpha_{j}=\right.$ $\left.1, \alpha_{j} \geq 0, \forall j\right\}$. For $x \in \mathbb{R}^{d}$ we denote $\|x\|_{Q}^{2}=x^{\top} Q x$ 


\section{PRELIMINARIES}

\section{A. Geometric prerequisites}

Consider first the definition of a support function [18], evaluated at $\eta \in \mathbb{R}^{d}$ for a given set $Q \subset \mathbb{R}^{d}$ :

$$
h_{Q}(\eta)=\sup _{q \in Q} \eta^{T} q .
$$

This notion has an interesting application for set inclusion validation. Let us consider two sets $X$ and $Y$, then the inclusion $X \subseteq Y$ holds only iff $h_{X}(\eta) \leq h_{Y}(\eta), \forall \eta \in \mathbb{R}^{d}$. Furthermore, if $Y$ is a polytope in the half-space form:

$$
Y=\left\{x \in \mathbb{R}^{d}: s_{i}^{\top} x \leq r_{i}, i=1 \ldots n_{v}\right\},
$$

then the previous inequality has to be checked only for a finite number of vectors, i.e. the condition becomes $h_{X}\left(s_{i}\right) \leq r_{i}, \forall i$. If $X$ is also a polytope described as convex combination of its extreme points:

$$
X=\left\{x \in \mathbb{R}^{d}: x=\sum \alpha_{j} v_{j}, \sum \alpha_{j}=1, \alpha_{j} \geq 0\right\},
$$

the inclusion condition is rewritten as $s_{i}^{\top} v_{j} \leq r_{i}, \forall i, j$.

We will use extensively zonotopes as a class of polytopes, endowed with a third representation due to their symmetry.

Definition 1 (Zonotopes-[19]): A zonotope is a centrally symmetric polytope, which can be described as a Minkowski sum of line segments. In its generator representation a zonotope $\mathcal{Z}(G, c)$ is described by center $c \in \mathbb{R}^{d}$ and generator matrix $G=\left[\begin{array}{lll}g_{1} & \cdots & g_{m}\end{array}\right] \in \mathbb{R}^{d \times m}$ :

$$
\mathcal{Z}(G, c)=\left\{c+\sum_{k=1}^{m} \xi_{k} g_{k}:\|\xi\|_{\infty} \leq 1\right\}
$$

Zonotopes own several properties of practical interest [20]:

i) are closed under Minkowski sum:

$$
\mathcal{Z}\left(G_{1}, c_{1}\right) \oplus \mathcal{Z}\left(G_{2}, c_{2}\right)=\mathcal{Z}\left(\left[\begin{array}{ll}
G_{1} & G_{2}
\end{array}\right], c_{1}+c_{2}\right) ;
$$

ii) are symmetric, up to their center:

$$
-\mathcal{Z}(G, c)=\mathcal{Z}(G,-c)
$$

iii) their volume has an explicit formulation [21]:

$$
\operatorname{Vol}(\mathcal{Z}(G, c))=\sum_{1 \leq j_{1}<j_{2} \ldots j_{d} \leq m}\left|\operatorname{det}\left(G^{j_{1} \ldots j_{d}}\right)\right|,
$$

where $G^{j_{1} \ldots j_{d}}$ denotes the matrix composed from columns of indices $j_{1} \ldots j_{d}$ from $G$.

Having the generator form of a zonotope, the half-space representation can be constructed as follows [14]: to each sequence of $d-1$ generators $1 \leq j_{1}<j_{2} \ldots j_{d-1} \leq m$ corresponds the pair $\left(h_{i}, k_{i}\right) \in \mathbb{R}^{d} \times \mathbb{R}$, where:

$$
h_{i} \perp g_{j_{l}}, \forall j_{l} \in\left\{j_{1} \ldots j_{d-1}\right\}, \quad k_{i}=\sum_{j_{l} \notin\left\{j_{1} \ldots j_{d-1}\right\}}\left|h_{i}^{\top} g_{j_{l}}\right| .
$$

Adding the center from generator representation, a zonotope is formulated in terms of its halfspaces (8) as:

$$
\mathcal{Z}(G, c)=\bigcap_{1 \leq j_{1}<\ldots j_{d-1} \leq m}\left\{x \in \mathbb{R}^{d}:\left|h_{i}(x-c)\right| \leq k_{i}\right\}
$$

Using the support functions in combination with the definition of a zonotope, the inclusion $\mathcal{Z}(G, c) \subseteq Y$, with $Y$ defined as in (2), is valid iff :

$$
s_{i}^{\top} c+\sum_{j}\left|s_{i}^{\top} g_{j}\right| \leq r_{i} \quad \forall i, j .
$$

Moreover, the inclusion of a polytopic set $X$, defined as in (3), into a zonotope $X \subseteq \mathcal{Z}(G, c)$ holds iff:

$$
\left|h_{i}^{\top}\left(v_{j}-c\right)\right| \leq k_{i} \quad \forall i, j .
$$

Consider a finite collection of hyperplanes from $\mathbb{R}^{d}$ :

$$
\mathbb{H}=\left\{\mathcal{H}_{i}\right\}_{i \in \mathcal{I}},
$$

with $\mathcal{H}_{i}=\left\{x \in \mathbb{R}^{d}: s_{i}^{\top} x=r_{i}\right\}$. Each of these hyperplanes divides the space in two disjoint regions:

$$
\begin{aligned}
& \mathcal{R}_{i}^{+}=\left\{x \in \mathbb{R}^{d}: \quad s_{i}^{\top} x \leq r_{i}\right\}, \\
& \mathcal{R}_{i}^{-}=\left\{x \in \mathbb{R}^{d}:-s_{i}^{\top} x \leq-r_{i}\right\} .
\end{aligned}
$$

Next, the space can be partitioned into cells using the hyperplane arrangement notion.

Definition 2 (Hyperplane arrangements - [22]): The collection $\mathbb{H}$ partitions the space into a union of disjoint cells $\mathcal{A}(\sigma)$, characterized by a sign tuple $\sigma \in\{-,+\}^{N}$ :

$$
\mathcal{A}(\sigma)=\bigcap_{i \in \mathcal{I}} \mathcal{R}_{i}^{\sigma(i)}
$$

The hyperplane arrangement of cells covering the entire space is described by the collection of all feasible sign tuples:

$$
\mathcal{A}(\mathbb{H})=\bigcup_{l=1 \ldots \gamma(N)} \mathcal{A}\left(\sigma_{l}\right),
$$

where $\sigma_{l} \in\{-,+\}^{N}$ is the sign tuple resulting from a nonempty intersection of half-spaces and $\gamma(N)$ is the number of feasible cells.

A (sub-)arrangement $\mathcal{B} \subseteq \mathcal{H}$ is called central if

$$
\bigcap_{H_{i} \in \mathcal{B}} H_{i} \neq \emptyset \text {. }
$$

Notation $\# \mathcal{B}$ denotes the number of hyperplanes and $\operatorname{rank}(\mathcal{B})$ the rank of the intersection.

Theorem 1 (Whitney's theorem - [23]): Let $\mathcal{A}$ be an arrangement in an $d$-dimensional space. Then the characteristic polynomial of $\mathcal{A}$ is defined as

$$
\chi_{\mathcal{A}}(t)=\sum_{\mathcal{B} \subseteq \mathcal{A}, \mathcal{B} \text { central }}(-1)^{\# \mathcal{B}} t^{d-\operatorname{rank}(\mathcal{B})} .
$$

Furthermore, the total number of regions and the number of bounded regions characterizing the arrangement are:

$$
\begin{aligned}
r(\mathcal{A}) & =(-1)^{d} \chi_{\mathcal{A}}(-1), \\
b(\mathcal{A}) & =(-1)^{\operatorname{rank}(\mathcal{A})} \chi_{\mathcal{A}}(1) .
\end{aligned}
$$

For a hyperplane arrangement with $n$ hyperplanes in general position $^{1}$, the bounds are therefore:

$$
r(\mathcal{A})=\sum_{k=0}^{d}\left(\begin{array}{l}
n \\
k
\end{array}\right), \quad b(\mathcal{A})=\left(\begin{array}{c}
n \\
d-1
\end{array}\right) .
$$

\footnotetext{
${ }^{1} \mathrm{An}$ arrangement is considered to be in general position iff no two hyperplanes are parallel (i.e., share the same normal).
} 


\section{B. Motivation}

Consider the collection of obstacles (blue regions in Fig. 1)

$$
\mathbb{P}=\bigcup_{j=1}^{N_{o}} P_{j}
$$

Gathering the collection of associated support hyperplanes defined as in (12) we reach the hyperplane arrangement (15). Labeling the feasible cells (14) into interdicted $\Sigma_{\mathbb{P}}=\{\sigma$ : $A(\sigma) \cap \mathbb{P} \neq \emptyset\}$ or allowed $\Sigma_{X \backslash \mathbb{P}}=\{\sigma: A(\sigma) \cap \mathbb{P}=\emptyset\}$ leads to a characterization of the feasible domain in which an agent moves (in order to avoid obstacle collisions). The feasible domain is characterized through a mixed-integer formulation further embedded into a constrained optimization strategy [9]. More precisely, a MPC (Model Predictive Control) strategy is employed in order to obtain a collision free trajectory for an agent described by LTI dynamics through a multi-obstacle environment:

$$
\begin{aligned}
\min _{\mathbf{u}}\left(\left\|x_{k+N_{p} \mid k}^{T}\right\|_{P}^{2}\right. & \left.+\sum_{l=1}^{N_{p}-1}\left\|x_{k+l \mid k}^{T}\right\|_{Q}^{2}+\sum_{l=1}^{N_{p}-1}\left\|u_{k+l \mid k}^{T}\right\|_{R}^{2}\right) \\
\text { s.t. } \quad x_{k+l \mid k} & =A x_{k+l-1 \mid k}+B u_{k+l-1 \mid k} \\
x_{k+l \mid k} & \in \mathcal{X}, u_{k+l \mid k} \in \mathcal{U} \\
x_{k+l \mid k} & \in \mathcal{C}_{\mathcal{X}}(\mathbb{P}) .
\end{aligned}
$$

The agent dynamics are described by (21a) with $x \in$ $\mathbb{R}^{d}$ the state vector, $u \in \mathbb{R}^{d_{u}}$ the input vector and the matrices $A, B$ of appropriate dimension. Furthermore, in (20) - (21) $N_{p}$ is the prediction horizon, the matrices $P$ (terminal cost penalty), $Q$ (output error penalty) and $R$ (control move penalty) are positive semi-definite and of appropriate dimensions. The sets $\mathcal{X}$ and $\mathcal{U}$ are compact sets from $\mathbb{R}^{d}$ and $\mathbb{R}^{d_{u}}$, respectively.

Let us consider in Fig. 1a a collection of obstacles as defined in (19) and delineate in Table I the number of:

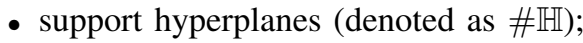

- feasible cells ${ }^{2}$ (denoted as $\gamma^{*}(N)$ ) with their offline constructing time (denoted as $t_{\gamma^{*}(N)}$ with $N=\# \mathbb{H}$ );

- interdicted cells (denoted as $\# \Sigma_{\mathbb{P}}$ ).

For further use, we delineate as well in Table I the parameters corresponding to the $3 \mathrm{D}$ case depicted in ${ }^{3}$ Fig. 1 b.

\begin{tabular}{|c|c|c|c|c|c|}
\hline & $\# \mathbb{P}$ & $\# \mathbb{H}$ & $\gamma^{*}(N)$ & $t_{\gamma^{*}(N)}[\mathrm{s}]$ & $\# \Sigma_{\mathbb{P}}$ \\
\hline$d=2$ & 7 & 34 & 419 & 9.22 & 75 \\
\hline$d=3$ & 10 & 106 & 57185 & 1255,88 & 1378 \\
\hline
\end{tabular}

TABLE I: Constructive parameters and computing time of the hyperplanes arrangements given in Fig. 1.

Several issues become apparent:

i) the complexity of the problem is strongly correlated with the number of cells in the arrangement $\left(\gamma^{*}(N)\right)$;

ii) the number of cells depends on the number of hyperplanes appearing in the obstacle description $(\# \mathbb{H})$.

\footnotetext{
${ }^{2}$ We take into account only the cells from the bounded domain $\mathcal{X}$.

${ }^{3}$ For clarity, we do not illustrate the hyperplanes in $3 \mathrm{D}$ case.
}

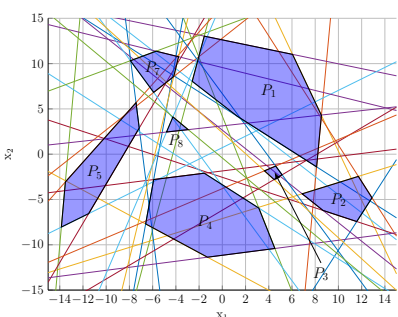

(a) $d=2$ and $\# H=7$

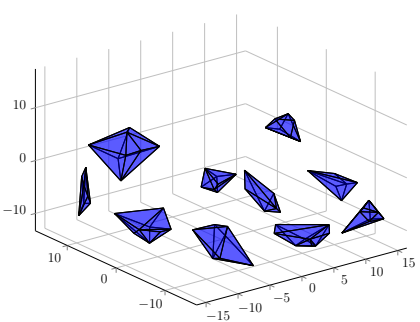

(b) $d=3$ and $\# H=10$
Fig. 1: Hyperplane arrangement for polytopic obstacles.

While the first issue comes naturally from the repetition of the feasible domain along the prediction horizon, the later one is particularly sensitive as a relatively small change in obstacle description leads to a potentially large increase in complexity. Herein lies the main motivation for the use of zonotopic approximations: instead of simply "accepting" the collection of obstacles (19) as it is, we proceed to overapproximate them via a pre-defined family of zonotopes in order to:

i) preserve a good approximation of the original interdicted domain (gauged via various measures);

ii) have a clear complexity evaluation (in terms of total number of cells) via hyperplane arrangement results;

Before the main developments we caution that a zonotope is centrally symmetric. Hence, not any convex shape can be rigorously approximated by an enclosing zonotope and this will be the main source of conservatism hereafter (and a potential direction of improvement). In particular, we notice that a symmetric approximation (zonotope) with a pre-defined topology (pre-imposed fixed direction of the generators) can be arbitrarily conservative. It is worth to be mentioned that a variety of algorithms provides overapproximations [13], [14] and many of them consider predefined families of zonotopes (e.g., by fixing the directions of the generators) and thus the present work can be useful for zonotopic approximation in general.

\section{CONTRIBUTIONS ON ZONOTOPIC APPROXIMATIONS}

Considering Def. 1 and (4) we refer to a family of zonotopes parametrized after their centers $c_{\ell} \in \mathbb{R}^{d}$ and scaling factors $\Delta_{\ell} \in \mathbb{R}^{m \times m}$ applied to a common generator "seed" (an a priori given matrix $G \in \mathbb{R}^{d \times m}$ ):

$$
\mathcal{Z}\left(G \Delta_{j}, c_{j}\right), \quad j=1 \ldots N_{o} .
$$

$\Delta_{\ell}$ is a diagonal matrix whose diagonal elements are equal $/$ distinct $^{4}$. The $\mathrm{k}$-th diagonal element is noted as $\delta_{j_{k}}$.

Having a common generator seed:

i) provides linear (in term of the scaling and center parameters) inclusion constraints;

ii) allows measures for the obstacle over-approximation (in terms of the $\ell_{1}, \ell_{\infty}$ norms and the volume);

\footnotetext{
${ }^{4}$ If not explicitly stated otherwise, we consider the later case since the former is a simplification of the later.
} 
iii) expresses explicitly the complexity of the representation (i.e., the number of cells of the associated arrangement).

\section{A. Set inclusions for parametrized zonotopes}

Replacing $g_{k}$ with $g_{k} \cdot \delta_{j_{k}}$ in (8), the half-space representation of the $\mathrm{j}$-th zonotope from (22) is given by:

$$
\begin{aligned}
h_{i} & \text { s.t. } h_{i} \perp g_{k}, \forall k \in\left\{k_{1} \ldots k_{d-1}\right\}, \\
k_{i}\left(\Delta_{j}\right) & =\sum_{k \notin\left\{k_{1} \ldots k_{d-1}\right\}}\left|h_{i}^{\top} g_{k}\right| \delta_{j_{k}},
\end{aligned}
$$

where $i$ enumerates the

$$
p(d, m)=\left(\begin{array}{c}
m \\
d-1
\end{array}\right)
$$

combinations of $d-1$ distinct generators selected from the list of $m$ available ones (i.e., $1 \leq k_{1}<\cdots<k_{d-1} \leq m$ ).

Remark 1: Note that $h_{i}$ remains unchanged with respect to (8) because the subspace perpendicular on $\left\{g_{k}\right\}_{k \in\left\{k_{1}, \ldots k_{d-1}\right\}}$ is identical with the one perpendicular on $\left\{g_{k} \cdot \delta_{j_{k}}\right\}_{k \in\left\{k_{1}, \ldots k_{d-1}\right\}}$, regardless of the $\delta_{j_{k}}$ values.

For further use we gather the support hyperplanes resulted from (23) into the collection:

$$
\tilde{\mathbb{H}}=\left\{\mathcal{H}\left(h_{i}, \pm k_{i}\left(\Delta_{j}\right)\right)\right\}, \forall j=1 \ldots N_{o}, i=1 \ldots p(d, m) .
$$

Using the parametrization (23), with sets $X, Y$ defined in (2)-(3), allows to reformulate the inclusion conditions (10), (11) into a linear form with respect to parameters $c_{j}, \Delta_{j}$ :

$$
\begin{aligned}
& \mathcal{Z}\left(G \Delta_{j}, c_{j}\right) \subseteq Y: s_{i}^{\top} c_{j}+\sum_{k=1}^{m}\left|s_{i}^{\top} g_{k}\right| \cdot \delta_{j_{k}} \leq r_{i}, \forall i, \\
& X \subseteq \mathcal{Z}\left(G \Delta_{j}, c_{j}\right):\left|h_{i}^{\top}\left(v_{k}-c_{j}\right)\right| \leq k_{i}\left(\Delta_{j}\right), \forall k .
\end{aligned}
$$

Remark 2: Using $X$ in its vertex representation (3) means that (26b) is translated into finding the tight enclosure of a set of points in $\mathbb{R}^{d}$ by a zonotope. [13] enforces a priori given points ( $v_{k}$ in (26b)) to stay on the zonotope boundary. Hence, the "inclusion" condition is written as:

$$
v_{k}=c_{j}+\sum_{i=1}^{m} \xi_{i j k} g_{i}, \quad-\delta_{j_{i}} \leq \xi_{i j k} \leq \delta_{j_{i}}, \forall i, k .
$$

The advantage of condition (27) is that it uses directly the generator representation but requires a large number of inequalities and is therefore not used here.

\section{B. Measures for zonotope approximations}

The overall goal is to provide adequate overapproximations (22) for the multi-obstacle environment (19). That is, seek a (inherently symmetric) zonotope $\mathcal{Z}\left(G \Delta_{j}, c_{j}\right)$ enclosing the known (usually non-symmetric) polytope $P_{j}$ such that a specific measure parametrized after $c_{j}, \Delta_{j}$ is minimized:

$$
\begin{aligned}
\left(\Delta_{j}, c_{j}\right)^{*}= & \arg \min _{\Delta_{j}, c_{j}} \mathcal{C}\left(\Delta_{j}, c_{j}\right) \\
& \text { s.t. } P_{j} \subseteq \mathcal{Z}\left(G \Delta_{j}, c_{j}\right) .
\end{aligned}
$$

In the following proposition we consider several measures.
Proposition 1: For the cost defined as in (28), the following measures ${ }^{5}$ are available:

i) zonotope volume $\operatorname{Vol}\left(\mathcal{Z}\left(G \Delta_{j}, c_{j}\right)\right)$ :

$$
\mathcal{C}\left(\Delta_{j}, c_{j}\right)=\sum_{1 \leq k_{1}<\cdots<k_{d} \leq m}\left|\operatorname{det}\left(G^{k_{1} \ldots k_{d}}\right)\right| \cdot \prod_{k \in\left\{k_{1}, \ldots, k_{d}\right\}} \delta_{j_{k}}
$$

ii) generator sum $\sum_{k=1}^{m} g_{k} \delta_{j_{k}}$ :

$$
\mathcal{C}\left(\Delta_{j}, c_{j}\right)=\left\|\Delta_{j}\right\|_{1}=\sum_{k=1}^{m} \delta_{j_{k}}
$$

iii) largest generator $\max _{k=1 \ldots m} g_{k} \delta_{j_{k}}$ :

$$
\mathcal{C}\left(\Delta_{j}, c_{j}\right)=\left\|\Delta_{j}\right\|_{\infty}=\max _{k=1 \ldots m} \delta_{j_{k}}
$$

Proof: See the Appendix.

Remark 3: Volume (29) is a sum of polynomial terms $\prod_{k \in\left\{k_{1}, \ldots, k_{d}\right\}} \delta_{j_{k}}$, thus, non-linear. Imposing equality among the scaling factors $\left(\delta_{j_{1}}=\ldots=\delta_{j_{m}}=\tilde{\delta}_{j}\right)$, leads to a simplified volume formulation:

$$
\operatorname{Vol}\left(\mathcal{Z}\left(G \Delta_{j}, c_{j}\right)\right)=\sum_{1 \leq k_{1}<\cdots<k_{d} \leq m}\left|\operatorname{det}\left(G^{k_{1} \ldots k_{d}}\right)\right| \cdot \tilde{\delta}_{j}^{d},
$$

which can be used instead of (29).

Solving (28) with (29) is burdensome in higher dimensions. This was the reason for considering the simpler (and less accurate) measures (30) - the Manhattan norm and (31) the infinity norm.

Remark 4: Taking the volume as the best measure for zonotope approximation, (29) is nonlinear but exact and (30), (31) are linear but coarse. Within this hierarchy is natural to consider the Hausdorff distance [22] as an intermediate measure (in terms of complexity and precision). This direction is not followed in this work.

\section{Hyperplane arrangement induced by the zonotopes}

Collection (25), based on (22) induces the hyperplane arrangement $^{6} \mathcal{A}(\tilde{\mathbb{H}})$. We aim to:

i) provide a cell count with explicit dependence on $m$, the number of generators and $N_{o}$, the number of obstacles; ii) compare it against the original arrangement $\mathcal{A}(\mathbb{H})$.

As shown in (25), the $2 \cdot p(d, m) \cdot N_{o}$ hyperplanes are partitioned in $2 N_{o}$ families: in each family there is a hyperplane with normal $h_{i}$ but with a different offset $\pm k_{i}\left(\Delta_{j}\right)$. This particularity allows the following proposition.

Proposition 2: $\mathcal{A}(\tilde{\mathbb{H}})$ has the characteristic polynomial:

$$
\chi(\mathcal{A})=\sum_{k=0}^{d}(-1)^{k} t^{d-k} \cdot\left(\begin{array}{c}
p(d, m) \\
k
\end{array}\right) \cdot\left(2 N_{o}\right)^{k}
$$

\footnotetext{
${ }^{5}$ Fulfilling the countable additivity, null-empty set and non-negativity properties over the class of zonotopes.

${ }^{6}$ For compactness, whenever clear from the context we use notation $\mathcal{A}$.
} 
to which correspond the following bounds:

$$
\begin{aligned}
& r(\mathcal{A})=\sum_{k=0}^{d}\left(\begin{array}{c}
p(d, m) \\
k
\end{array}\right) \cdot\left(2 N_{o}\right)^{k}, \\
& b(\mathcal{A})=\left|\sum_{k=0}^{d}(-1)^{k}\left(\begin{array}{c}
p(d, m) \\
k
\end{array}\right) \cdot\left(2 N_{o}\right)^{k}\right| .
\end{aligned}
$$

Proof: See the Appendix.

These results allow to further derive a bound for the number of generators in the zonotopic representation.

Corollary 1: Assuming $n_{o}^{*}$ support hyperplanes in (19), for any $m \in \mathbb{N}^{+}$which verifies:

$$
\sum_{k=0}^{d}\left(\begin{array}{c}
p(d, m) \\
k
\end{array}\right) \cdot\left(2 N_{o}\right)^{k} \leq \sum_{k=0}^{d}\left(\begin{array}{c}
n_{o}^{*} \\
k
\end{array}\right)
$$

the arrangement $\mathcal{A}(\tilde{\mathbb{H}})$ has fewer cells than $\mathcal{A}(\mathbb{H})$.

Proof: The left-side of (35) comes from (34) and the right side from (18) with $n \mapsto n_{o}^{*}$.

While the previous results hold for $\mathbb{R}^{d}$, it is worthwhile to particularize them for $d=3$.

Corollary 2: For the case $d=3$, we have that:

i) the total and the bounded number of cells for $\mathcal{A}(\tilde{\mathbb{H}})$ is:

$$
\begin{array}{r}
r(\mathcal{A})=1+\eta \cdot 2 N_{o}+\eta(\eta-1) \cdot 2 N_{o}^{2} \\
+\quad \frac{1}{6} \eta(\eta-1)(\eta-2) 8 N_{o}^{3}, \\
b(\mathcal{A})=\mid 1-\eta \cdot 2 N_{o}+\eta(\eta-1) \cdot 2 N_{o}^{2} \\
-\quad \frac{\eta(\eta-1)(\eta-2)}{6} 8 N_{o}^{3} \mid ;
\end{array}
$$

where ${ }^{7} \eta=\frac{1}{2} m(m-1)$;

ii) the largest $m$ s.t. $\mathcal{A}(\tilde{\mathbb{H}})$ has fewer cells than $\mathcal{A}(\mathbb{H})$ is :

$$
m=\left\lfloor\frac{1+\sqrt{1+8 \eta^{*}}}{2}\right\rfloor .
$$

where $\eta^{*}$ represents the real solution of a solvable third order equation ${ }^{8}$.

Proof: See the Appendix.

Let us revisit the previous illustrative examples and proceed to find the zonotopic over-approximations of the obstacles described in Fig. 1. The simulations were done for different variants of the generator seed $G$ in (22) for both $d=2$ and $d=3$ dimensions:

$$
\begin{gathered}
G_{1,2,3} \in\left\{\left[\begin{array}{lll}
1 & 0 & 1 \\
0 & 1 & 1
\end{array}\right],\left[\begin{array}{ll}
1 & 0 \\
0 & 1
\end{array}\right],\left[\begin{array}{ccc}
-1 & 0 & -1 \\
0 & 1 & 1
\end{array}\right]\right\}, \\
G_{4,5} \in\left\{\left[\begin{array}{lll}
1 & 0 & 0 \\
0 & 1 & 0 \\
0 & 0 & 1
\end{array}\right],\left[\begin{array}{llll}
1 & 0 & 0 & 1 \\
0 & 1 & 0 & 1 \\
0 & 0 & 1 & 1
\end{array}\right]\right\} .
\end{gathered}
$$

Maintaining the structure of Table I, we delineate in Table II some parameters of interest. Thus, we indicate the total

\footnotetext{
${ }^{7}$ Also, the particularization can be done for the case $d=2$. In (36) $\eta$ is replaced by $m$ (because of $p(2, m)=\left(\begin{array}{c}m \\ 1\end{array}\right)=m$ ) and, in both $r(\mathcal{A})$ and $b(\mathcal{A})$ the last term is eliminated.

${ }^{8}$ For the largest $m$ in (37) for the case $d=2$, we only need to solve a second order equation, holding the positive root, hence: $m=$ $\left\lfloor\frac{\left(N_{o}-1\right)+\sqrt{\left(N_{o}-1\right)^{2}+n_{o}^{*}\left(n_{O}^{*}+1\right)}}{2 N_{o}}\right\rfloor$.
}

computing time corresponding to each considered method $\left(t_{s o l}\right)$, the relative modification of the number of cells $\left(\frac{\Delta \gamma(N)}{\gamma(N)}=\frac{\left.\gamma^{*}(N)-\gamma(N)\right)}{\gamma(N)} \cdot 100\right)$ and a couple volume specifications: the volume of the over-approximation $(V)$ and the relative error with respect to the volume of the polytopic obstacles $\left(\frac{\Delta V}{V}=\frac{\operatorname{Vol}(\mathbb{Z})-\operatorname{Vol}(\mathbb{P})}{\operatorname{Vol}} \cdot 100\right)$.

We note that $\|\cdot\|_{1}$ has a better behavior than $\|\cdot\|_{\infty}$ (i.e., its relative volume error is smaller) and that the volume criterion gives the most accurate approximation (at the price of a large computational effort). We conclude thus that $\|\cdot\|_{1}$ provides the best compromise between over-approximation error and computation time. Additionally, we observe an inverse proportionality between the volume of the approximation and number of hyperplanes (and implicitly the number of cells). Note that this factor can be tweaked by a suitable choice of the generator matrix $G$. Lastly, as illustrated in Fig. 2, the choice of the matrix seed $G$ is not trivial and strongly depends on the shapes of the obstacles considered (the choice will impact the over-approximations).

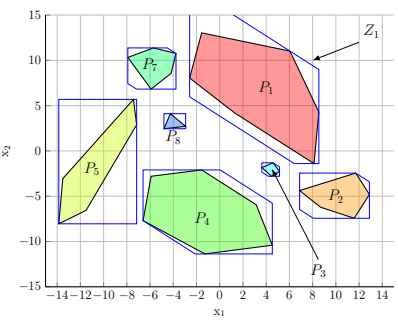

(a) Volume with $G=G_{3}$.

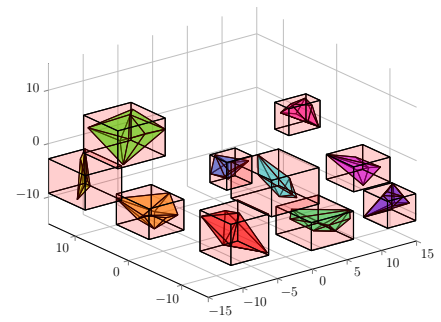

(b) Volume with $G=G_{4}$.
Fig. 2: Zonotopic approximation for the multi-obstacle environment from Fig. 1.

While for $d=2$ the results are somewhat ambiguous (the decrease of the complexity is not marked), for the $d=3$ case the impact is substantial on the number of cells. We remark a significant decrease of this number, even though the choice of the matrix $G$ was empirical and not the result of an optimization process. Moreover, the cell merging procedure [10] is burdensome for the polytopic representation (Fig. 1b) but it is a usable technique for the zonotopic over-approximation. On the other hand, there are no improvements regarding the volume and that highlights the influence of the "common seed".

\section{Multi-Obstacle Collision aVoidance}

Let us consider the following agent dynamics (21a) in $\mathbb{R}^{d}$ :

$$
A=\left[\begin{array}{cc}
O_{d} & I_{d} \\
O_{d} & -\frac{\mu}{M} I_{d}
\end{array}\right], \quad B=\left[\begin{array}{c}
O_{d} \\
M I_{d}
\end{array}\right],
$$

where $\mu=3$ and $M=60$. The agent's state and input are constrained: $\mathcal{X}=\left\{x:-15 \leq x_{i} \leq 15, \forall i=1 \ldots 2 d\right\}$ and $\mathcal{U}=\left\{u:-1 \leq u_{i} \leq 1, \forall i=1 \ldots 2 d\right\}$.

\section{A. Optimization-based control}

The constrained optimization problem (20) has to be solved over a non-convex domain $C_{\mathcal{X}}(\mathbb{P})$ in $(21 \mathrm{c})$. Thus, 


\begin{tabular}{|c|c|c|c|c|c|c|c|c|c|c|c|}
\hline & Measure & Constraints & G & $t_{\text {sol }}$ & $\# \mathbb{H}$ & $\gamma^{*}(N)$ & $\frac{\Delta \gamma(N)}{\gamma(N)}(\%)$ & $t_{\gamma^{*}(N)}$ & $\# \Sigma_{\mathbb{P}}$ & $\mathrm{V}$ & $\frac{\Delta V}{V}(\%)$ \\
\hline \multirow{12}{*}{$d=2$} & \multirow{3}{*}{$\|\delta\|_{1}$} & \multirow{3}{*}{ (26) } & $G_{1}$ & 8,13 & 42 & 505 & 20,53 & 9,53 & 197 & 376,98 & 71,7 \\
\hline & & & $G_{2}$ & 8,02 & 28 & 225 & $-46,30$ & 3,81 & 101 & 410,07 & 86,78 \\
\hline & & & $G_{3}$ & 8,27 & 42 & 534 & 27,45 & 10,09 & 167 & 368,93 & 68,04 \\
\hline & \multirow{3}{*}{$\|\delta\|_{\infty}$} & \multirow{3}{*}{ (26) } & $G_{1}$ & 8,19 & 42 & 441 & 5,25 & 8,19 & 374 & 897,92 & 308,98 \\
\hline & & & $G_{2}$ & 8,01 & 28 & 225 & $-46,30$ & 3,91 & 175 & 583,33 & 165,69 \\
\hline & & & $G_{3}$ & 8,19 & 42 & 441 & 5,25 & 8,19 & 374 & 897,92 & 308,98 \\
\hline & \multirow{3}{*}{ Vol } & \multirow{3}{*}{ (26) } & $G_{1}$ & 9,40 & 42 & 510 & 21,72 & 9,66 & 199 & 368,41 & 67,8 \\
\hline & & & $G_{2}$ & 9,19 & 28 & 225 & $-46,30$ & 3,75 & 101 & 410,07 & 86,78 \\
\hline & & & $G_{3}$ & 9,30 & 40 & 530 & 26,49 & 10,27 & 169 & 374,99 & 70,8 \\
\hline & \multirow{3}{*}{ Vol } & \multirow{3}{*}{$\begin{array}{l}(26)+ \\
\delta_{j}=\delta\end{array}$} & $G_{1}$ & 9,08 & 42 & 479 & 14,32 & 9,94 & 481 & 897,92 & 308,98 \\
\hline & & & $G_{2}$ & 8,88 & 28 & 225 & $-46,30$ & 3,84 & 193 & 583,33 & 165,69 \\
\hline & & & $G_{3}$ & 9,05 & 42 & 531 & 26,73 & 11,28 & 272 & 628,72 & 186,37 \\
\hline \multirow{8}{*}{$d=3$} & \multirow{2}{*}{$\|\delta\|_{1}$} & \multirow{2}{*}{ (26) } & $G_{4}$ & 9,82 & 60 & 8400 & $-85,31$ & 105,98 & 934 & 1857,46 & 323,56 \\
\hline & & & $G_{5}$ & 10,50 & 120 & 62480 & 9,26 & 1145,09 & 4952 & 2019,34 & 360,47 \\
\hline & \multirow{2}{*}{$\|\delta\|_{\infty}$} & \multirow{2}{*}{ (26) } & $G_{4}$ & 9,71 & 60 & 8000 & $-86,01$ & 100,22 & 1127 & 2623,3 & 498,19 \\
\hline & & & $G_{5}$ & 10,59 & 120 & 51396 & $-10,12$ & 932,12 & 9432 & 5852,55 & 1234,56 \\
\hline & \multirow{2}{*}{ Vol } & \multirow{2}{*}{ (26) } & $G_{4}$ & 11,02 & 60 & 8400 & $-85,31$ & 105,07 & 934 & 1857,46 & 323,56 \\
\hline & & & $G_{5}$ & 11,79 & 84 & 24528 & $-57,11$ & 413,07 & 2218 & 1908,2 & 335,13 \\
\hline & \multirow{2}{*}{ Vol } & \multirow{2}{*}{$\begin{array}{l}(26)+ \\
\delta_{j}=\delta\end{array}$} & $G_{4}$ & 10,49 & 60 & 7220 & $-87,37$ & 90,54 & 1124 & 2623,3 & 498,19 \\
\hline & & & $G_{5}$ & 11,07 & 120 & 49200 & $-13,96$ & 867,08 & 9436 & 5852,55 & 1234,56 \\
\hline
\end{tabular}

TABLE II: Performance of the zonotopic over-approximation techniques.

$C_{\mathcal{X}}(\mathbb{P})$ should be described in an efficient manner. As was stated in [9] the use of half-spaces and of hyperplane arrangement with a mixed-integer formalism leads to effective control strategies. Hereinafter, we aim to validate the benefits brought by the use of the parametrized zonotopic sets.

Using (20), we compare the performances of the control strategy corresponding to a polytopic representation (as in Fig. 1) to a zonotopic over-approximation (we assume no overlaps). We depict in Fig. 3 the agent motion starting from the same initial position in the multi-obstacle environment (Fig. 1a) and over-approximated (Fig. 2a).

\begin{tabular}{|l|c|c|c|c|}
\hline & Topology & $N_{\text {goal }}$ & $t_{\text {goal }}(\mathrm{sec})$ & $t_{\text {worst }}(\mathrm{sec})$ \\
\hline \multirow{2}{*}{$d=2$} & $\mathbb{P}$ & 143 & 11.64 & 0.22 \\
\cline { 2 - 5 } & $\mathbb{Z}$ & 146 & 10.07 & 0.18 \\
\hline \multirow{2}{*}{$d=3$} & $\mathbb{P}$ & 98 & 83.87 & 0.81 \\
\cline { 2 - 5 } & $\mathbb{Z}$ & 132 & 57.07 & 0.42 \\
\hline
\end{tabular}

TABLE III: Simulation results for (20) with $N_{p}=10, P=$ $10 I_{2 d}, Q=I_{2 d}, R=I_{d}$.

In Table III we delineate some noteworthy computational characteristics: $N_{\text {goal }}$ represents the number of steps necessary to attain a neighborhood of the final destination, $t_{\text {goal }}$ the total time neccesary to compute the trajectory, and $t_{\text {worst }}$ stands for the worst time necessary to solve (20).

We note that for $d=2$ case the computational performances are quasi-similar for both representations. We have a longer trajectory for the zonotopic representation, as was expected, because of the additional "space" $\left(P_{j} \backslash \mathcal{Z}\left(G \Delta_{j}, c_{j}\right)\right)$ which becomes an interdicted region. However, $t_{\text {goal }}$ is smaller due to the symmetry properties while $t_{w o r s t}$ is not significantly changed. The last mentioned aspect is directly caused by the values presented in Table II - the number of cells. Furthermore, for the $d=3$ case the differences are more noticeable. We have a reduction of $70 \%$ of the $t_{\text {goal }}$, and $t_{\text {worst }}$ is reduced at half for the zonotopic representation.

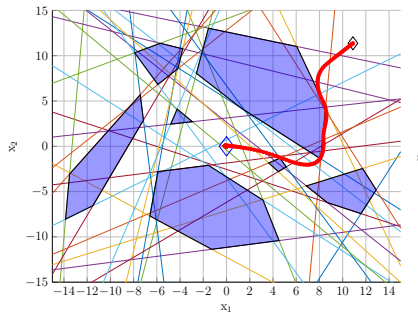

(a) Polytopes

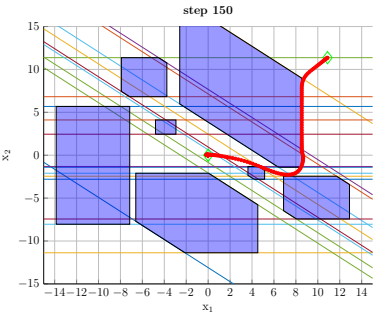

(b) Zonotopes
Fig. 3: Illustration of obstacle avoidance for $d=2$.

A reasonable assumption (confirmed by the simulations) is that an increase of the prediction horizon $N_{p}$ will accentuate the differences between these two representations but may also lead to inapplicable control strategies due to the computational effort. Moreover, an optimized selection of the common "seed" will bring a more prominent difference without an undesirable effect on the control algorithm.

\section{B. Sample/Graph-based approaches}

In contrast to the MIP approach where the discrete decisions are encoded in a mathematical formalism and are solved as such, the graph-based approaches reduce these discrete decision to the search of the shortest path between nodes in a graph. The PRM (probabilistic roadmaps) is a multiple-query method in the sense that after the construction of the roadmap (a rich set of feasible paths) it answers queries by computing an optimal path through the graph. The PRM is an useful method if an awareness map of the environment is available [6]. Hence, a comparison with the MIP-based approach is pertinent. 
There exist many variants for the PRM, each representing a valuable improvement, but for this study we select the classical PRM. Thus, we use a method based on visibility [24]. Specifically, we randomly select a number of samples within the workspace, we keep only the ones from the nonconvex feasible domain and we connect these points based on a visibility criterion (if there is a line connecting the points without intersecting any obstacle, then the points are visible from one another). Having this visibility graph (offline computed, assuming the existence of the awareness map), we only link the start and the final position to the nearest graph nodes and find the shortest path through the graph, e.g., using Dijkstra algorithm [6]. Afterward, we use a tracking algorithm in order to follow the obtained path (the black lines from Fig. 4). We opted to use MPC for tracking ${ }^{9}$, the problem (20) without the constraint (21c) for each segment of the path (maintaining the same values of the MPC parameters as in Table III).

\begin{tabular}{|c|c|c|c|c|}
\hline & Approach & $N_{\text {goal }}$ & $t_{\text {goal }}(\mathrm{sec})$ & $\ell_{t}(\mathrm{~m})$ \\
\hline \multirow{2}{*}{$d=2$} & MIP $(\mathbb{Z})$ & 146 & 10.07 & 21.36 \\
\cline { 2 - 5 } & PRM & 172 & 9.81 & 24.41 \\
\hline \multirow{2}{*}{$d=3$} & MIP $(\mathbb{Z})$ & 132 & 57.07 & 25.57 \\
\cline { 2 - 5 } & PRM & 192 & 12.15 & 29.96 \\
\hline
\end{tabular}

TABLE IV: Results for the agent trajectories in Fig. 4.

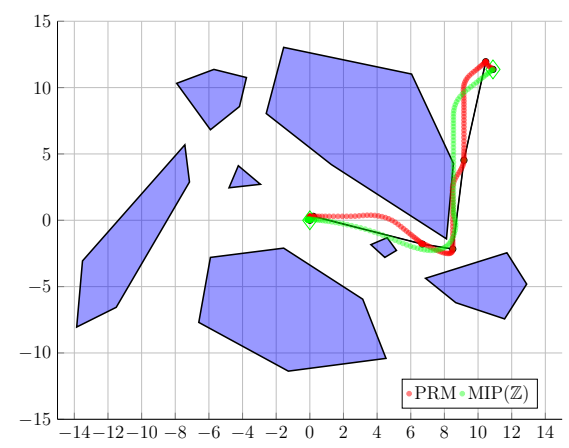

Fig. 4: Illustration of obstacle avoidance for $d=2$.

As it can be noted from the results depicted in Table IV, the trajectory length $\left(\ell_{t}\right)$ obtained with PRM is longer than the one corresponding to the MIP formulation with zonotopic representation. As well we observe that $t_{\text {goal }}$ corresponding to the PRM method does not depend on the value of $d$, whereas the zonotopic representation is strongly impacted by the space dimension. Within these two examples ${ }^{10}$, the MIP-based approach performs better in the sense of number of steps and trajectory length. Nevertheless, the choice of the approach needs always an application-dependent analysis. However, the zonotope approximation impact is clearly exemplified in this study and provides manageable complexity and structural properties. These last elements can be crucial in the certification of control algorithms.

\footnotetext{
${ }^{9}$ Note that the performances of the PRM method strongly depend on the choice of the tracking algorithm.

${ }^{10}$ We illustrate the trajectories only for $d=2$ case for reasons of space and visibility.
}

\section{CONCLUSIONS}

This work presented a geometric view for the collision avoidance problem using zonotopic over-approximations of the obstacles. We emphasized the benefits of choosing a particular family of sets (parametrized zonotopes) regarding the complexity of a non-convex feasible domain representation. Additionally, we compared the MPC-based collision avoidance with the heuristic PRM (Probabilistic Roadmaps) technique and confirmed the potential advantages.

In this paper we concentrate exclusively on overapproximation aspect and on its effects on the optimization problem. We have neglected the possibility of the overlapping of some of the approximations and we have not tackled issues like lost or narrowing of the passages among the obstacles. This will be the topic of further research and it will present that separation conditions can be imposed in order to maintain passages among the obstacles.

\section{APPENDIX}

\section{Proof of Proposition 1}

i) Replacing $g_{k}$ with $g_{k} \cdot \delta_{j_{k}}$ in (7) leads to

$$
\operatorname{Vol}\left(\mathcal{Z}\left(G \Delta_{j}, c_{j}\right)\right)=\sum_{1 \leq k_{1}<\cdots<k_{d} \leq m}\left|\operatorname{det}\left(G^{k_{1} \cdots k_{d}} \cdot \Delta_{j}^{k_{1} \ldots k_{d}}\right)\right| .
$$

Noting that $\operatorname{det}(M \cdot N)=\operatorname{det}(M) \cdot \operatorname{det}(N)$ and that $\Delta_{j}$ is a diagonal matrix with positive elements gives (29).

ii) Assuming without loss of generality that $\left\|g_{k}\right\|_{1}=1$, the cost reduces immediately to (30).

iii) Similar reasoning with case ii), assuming $\left\|g_{k}\right\|_{\infty}=1$.

\section{Proof of Proposition 2}

To apply Whitney's theorem and obtain the bounds (18), the key is to count how many central arrangements of rank $k$ there are. Hence, we need to avoid selecting parallel hyperplanes from the available $p(d, m) \cdot 2 N_{o}$. We consider each of the $2 N_{o}$ families of $p(d, m)$ hyperplanes and select as follows: from the 1 st family we select $j_{1}^{-}$; in the 2 nd family there remain $m-j_{1}^{-}$available (the others are parallel to those already selected and thus unsuitable) from which we select $j_{1}^{+}$; the procedure continues up to the $2 N_{0}$-th family from which we select $j_{N_{o}}^{+}$.

Enumerating all sequences $j_{1}^{-}, \ldots, j_{N_{o}}^{+}$which add to $k$ and taking all the possible combinations for a fixed sequence provides the total number of central arrangements of rank $k$ :

$$
\begin{aligned}
\sum_{j_{1}^{-}+j_{1}^{+}+\cdots+j_{n_{o}}^{-}+j_{n_{o}=k}^{+}}\left(\begin{array}{c}
p \\
j_{1}^{-}
\end{array}\right) \cdot\left(\begin{array}{c}
p-j_{1}^{-} \\
j_{1}^{+}
\end{array}\right) \\
\ldots\left(\begin{array}{c}
p-j_{1}^{-}-j_{1}^{+}-\cdots-j_{N_{o}}^{-} \\
j_{N_{o}}^{+}
\end{array}\right) .
\end{aligned}
$$

Writing explicitly the combinatorial terms for a sequence 


$$
\begin{aligned}
& j_{1}^{-}, j_{1}^{+} \ldots, j_{N_{o}}^{-}, j_{N_{o}}^{+} \\
& \frac{p !}{j_{1}^{-} ! \cdot\left(p-j_{1}^{-}\right) !} \cdot \ldots \cdot \frac{\left(p-j_{1}^{-} \cdots-j_{n_{o}}^{-}\right) !}{j_{n_{o}}^{+} ! \cdot\left(p-j_{1}^{-} \ldots j_{N_{o}}^{-}-j_{N_{o}}^{+}\right) !} \\
&=\frac{k !}{(p-k) ! \cdot k !} \cdot \frac{k !}{j_{1}^{-} ! \cdot j_{1}^{+} ! \cdot \ldots \cdot j_{N_{o}}^{-} ! \cdot j_{N_{o}}^{+} !} \\
&=\left(\begin{array}{l}
p \\
k
\end{array}\right) \cdot \frac{k !}{j_{1}^{-} ! \cdot j_{1}^{+} ! \cdot \ldots \cdot j_{N_{o}}^{-} ! \cdot j_{N_{o}}^{+} !}
\end{aligned}
$$

and identifying $\frac{k !}{j_{1}^{-} ! \cdot j_{1}^{+} ! \ldots j_{N_{o}}^{-} ! \cdot j_{N_{o}}^{+} !}$as the coefficient from the multinomial theorem [25] allows to rewrite (40) as

$$
\sum_{j_{1}^{-}+\ldots j_{N_{o}}^{+}}\left(\begin{array}{c}
p \\
k
\end{array}\right) \cdot \frac{k !}{j_{1}^{-} ! \cdot j_{1}^{+} ! \ldots j_{N_{o}}^{-} ! \cdot j_{N_{o}}^{+} !}=\left(\begin{array}{l}
p \\
k
\end{array}\right) \cdot\left(2 N_{o}\right)^{k} .
$$

Introducing (41) in (16) leads directly to (33) which, applied as in (17), leads to (34), thus concluding the proof.

\section{Proof of Corollary 2}

For $d=3$, the number of support hyperplanes for a zonotope becomes $p(3, m)=\left(\begin{array}{c}m \\ 2\end{array}\right)=\frac{1}{2} m(m-1)$.

i) Introducing $p(3, m)$ in (34) directly leads to (36).

ii) Introducing $p(3, m)$ in $(35)$ reduces it to a third-order inequality in $\eta$ as it follows:

$$
\begin{aligned}
& 2\left(8 N_{0}^{3}-12 N_{0}^{2}+12 N_{0}\right) \eta-3\left(8 N_{0}^{3}-4 N_{0}^{2}\right) \eta^{2}+8 N_{0}^{3} \eta^{3} \leq \\
& \leq n_{o}^{*}+\frac{1}{6} n_{o}^{*}\left(n_{o}^{*}-1\right)\left(n_{o}^{*}+1\right) .
\end{aligned}
$$

Using the notation $\lambda$ for the right term of (42) we rewrite it as:

$$
8 N_{0}^{3} \eta^{3}-12 N_{0}^{2}\left(2 N_{0}-1\right) \eta^{2}+8 N_{0}\left(2 N_{0}^{2}-3 N_{0}+3\right)-\lambda \leq 0 .
$$

Therefore, we can compute the discriminant:

$$
\begin{aligned}
\Delta= & N_{0}^{6} \quad\left[-359424-34560 \lambda-1728 \lambda^{2}+\right. \\
+(829440 & +82944 \lambda) N_{0}-(1022976+27648 \lambda) N_{0}^{2} \\
& \left.+663552 N_{0}^{3}-221184 N_{0}^{4}+16384 N_{0}^{6}\right] .
\end{aligned}
$$

We note that $\Delta$ is negative for the so-defined positive values of $N_{0}$ and $\lambda$. This means that the third order equation has one real solution and two complex ones. The computing of them involves calculating:

$$
\begin{aligned}
\Delta_{0} & =N_{0}^{4}\left(-432+192 N_{0}^{2}\right), \\
\Delta_{1} & =N_{0}^{6}\left(-17280-1728 \lambda+41472 N_{0}-13824 N_{0}^{2}\right), \\
C & =\sqrt[3]{\frac{\Delta_{1} \pm \sqrt{1728 N_{0}^{2} \Delta}}{2}} .
\end{aligned}
$$

Thus, the real solution is given by:

$$
\eta^{*}=\frac{1}{24 N_{0}^{3}}\left[12 N_{0}^{2}\left(2 N_{0}-1\right)+C+\frac{\Delta_{0}}{C}\right] .
$$

This real solution is introduced in the $\eta$ definition, obtaining a second-order equation in $m$. This inequality has two real solutions (one negative and one positive) which means that the largest $m$ verifying the inequality is the closest (at its left) from the positive solution, hence, (37) .

\section{REFERENCES}

[1] F. Janecek, M. Klauco, M. Kaluz, and M. Kvasnica, "OPTIPLAN: A Matlab Toolbox for Model Predictive Control with Obstacle Avoidance," IFAC-PapersOnLine, vol. 50, no. 1, pp. 531-536, Jul. 2017.

[2] A. Richards and J. P. How, "Aircraft trajectory planning with collision avoidance using mixed integer linear programming," in American Control Conference, 2002. Proceedings of the 2002, vol. 3. IEEE, 2002, pp. 1936-1941.

[3] F. Stoican, I. Prodan, and S. Olaru, "Hyperplane arrangements in mixed-integer programming techniques. Collision avoidance application with zonotopic sets," in 2013 European Control Conference (ECC), Jul. 2013, pp. 3155-3160.

[4] Y.-b. Chen, G.-c. Luo, Y.-s. Mei, J.-q. Yu, and X.-1. Su, "UAV path planning using artificial potential field method updated by optimal control theory," International Journal of Systems Science, vol. 47, no. 6, pp. 1407-1420, Apr. 2016.

[5] R. Olfati-Saber and R. M. Murray, "Distributed cooperative control of multiple vehicle formations using structural potential functions," in IFAC world congress, vol. 15, no. 1, 2002, pp. 242-248.

[6] S. Karaman and E. Frazzoli, "Sampling-based algorithms for optimal motion planning," The International Journal of Robotics Research, vol. 30, no. 7, pp. 846-894, Jun. 2011.

[7] S. M. LaValle, "Rapidly-exploring random trees: A new tool for path planning," Tech. Rep., 1998.

[8] J.-C. Latombe, Robot motion planning. Springer Science \& Business Media, 2012, vol. 124.

[9] I. Prodan, F. Stoican, S. Olaru, and S.-I. Niculescu, "Mixed-Integer Representations," in Mixed-Integer Representations in Control Design, ser. SpringerBriefs,Engineering. Springer, Cham, 2016.

[10] - "Enhancements on the hyperplanes arrangements in mixedinteger programming techniques," Journal of Optimization Theory and Applications, vol. 154, no. 2, pp. 549-572, 2012.

[11] J. P. Vielma and G. L. Nemhauser, "Modeling disjunctive constraints with a logarithmic number of binary variables and constraints," Mathematical Programming, vol. 128, no. 1-2, pp. 49-72, 2011.

[12] L. Jaulin, M. Kieffer, O. Didrit, and E. Walter, Applied interval analysis: with examples in parameter and state estimation, robust control and robotics. Springer Science \& Business Media, 2001, vol. 1.

[13] L. J. Guibas, A. Nguyen, and L. Zhang, "Zonotopes As Bounding Volumes," in Proceedings of the Fourteenth Annual ACM-SIAM Symposium on Discrete Algorithms, ser. SODA '03. Philadelphia, PA, USA: SIAM, 2003, pp. 803-812.

[14] M. Althoff, O. Stursberg, and M. Buss, "Computing reachable sets of hybrid systems using a combination of zonotopes and polytopes," Nonlinear analysis: hybrid systems, vol. 4, no. 2, pp. 233-249, 2010.

[15] A. Ingimundarson, J. M. Bravo, V. Puig, T. Alamo, and P. Guerra, "Robust fault detection using zonotope-based set-membership consistency test," International journal of adaptive control and signal processing, vol. 23, no. 4, pp. 311-330, 2009.

[16] T. Alamo, J. M. Bravo, and E. F. Camacho, "Guaranteed state estimation by zonotopes," Automatica, vol. 41, no. 6, pp. 1035-1043.

[17] M. Althoff, "Cora 2015 manual," TU Munich, vol. 85748, 2015.

[18] I. Kolmanovsky and E. G. Gilbert, "Theory and computation of disturbance invariant sets for discrete-time linear systems," Mathematical problems in engineering, vol. 4, no. 4, pp. 317-367, 1998.

[19] W. Kuhn, "Rigorously computed orbits of dynamical systems without the wrapping effect," Computing, vol. 61, no. 1, pp. 47-67, Mar. 1998.

[20] K. Fukuda, "From the zonotope construction to the Minkowski addition of convex polytopes," Journal of Symbolic Computation, vol. 38, pp. 1261-1272, Oct. 2004.

[21] E. Gover and N. Krikorian, "Determinants and the volumes of parallelotopes and zonotopes," Linear Algebra and its Applications, vol. 433, no. 1, pp. 28-40, Jul. 2010.

[22] G. M. Ziegler, Lectures on polytopes. Springer Science \& Business Media, 2012, vol. 152.

[23] R. P. Stanley, "An introduction to hyperplane arrangements," in Lecture notes, IAS/Park City Mathematics Institute, 2004.

[24] T. Lozano-Pérez and M. A. Wesley, "An algorithm for planning collision-free paths among polyhedral obstacles," Communications of the ACM, vol. 22, no. 10, pp. 560-570, 1979.

[25] M. Hazewinkel, Multinomial coefficient, Encyclopedia of Mathematics. Springer, 2001. 